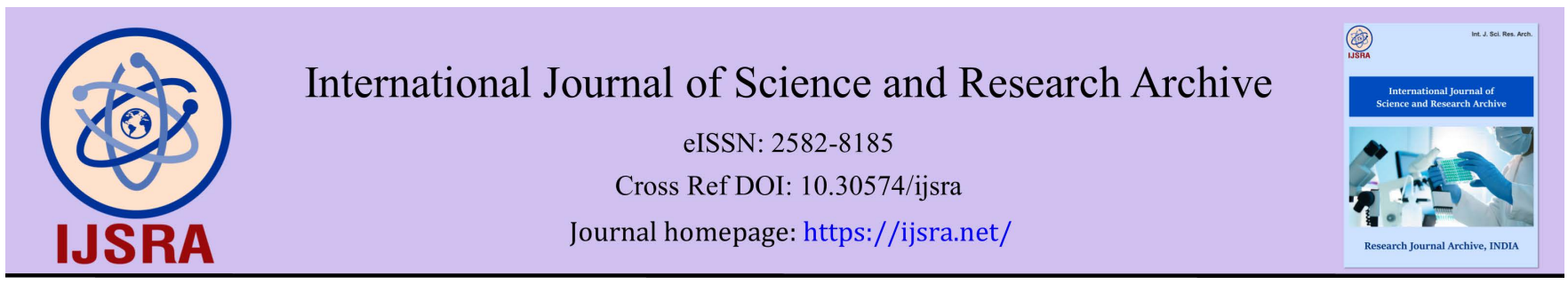

(RESEARCH ARTICLE)

\title{
Antimicrobial activity of selected aminoglycoside and fluoroquinolone eye drops against Staphylococcus aureus isolated from the conjunctiva of a bacterial conjunctivitis patient
}

Chigozie John Ekenze 1, Oyinyechi Lilian Umunnakwe 2, Timothy Olugbenga Ogundeko ${ }^{3,}{ }^{*}$, Chioma Lilian Owunna ${ }^{4}$, Florence Akuchi Mbatuegwu ${ }^{5}$ and Mamzhil Seljul Crown Ramyil 6

${ }^{1}$ Department of Optometry, Imo State University, Owerri Nigeria.

${ }^{2}$ Department of Optometry, Federal University of Technology, Owerri Nigeria.

${ }^{3}$ Department of Pharmacology and Therapeutics, College of Medicine and Health Sciences, Bingham University, Jos Campus Nigeria.

${ }^{4}$ Department of Optometry, Bingham University Teaching Hospital, Jos Nigeria.

${ }^{5}$ Department of Optometry, College of Medicine and Health Sciences, Bingham University, Karu Nasarawa Nigeria.

${ }^{6}$ Department of Microbiology and Parasitology, College of Medicine and Health Sciences, Bingham University, Jos Campus Nigeria.

International Journal of Science and Research Archive, 2021, 02(02), 151-157

Publication history: Received on 15 April 2021; revised on 20 May 2021; accepted on 23 May 2021

Article DOI: https://doi.org/10.30574/ijsra.2021.2.2.0072

\begin{abstract}
A comparative in vitro study was conducted to ascertain the efficacy of 4 selected antibiotic eye drops against Staphylococcus aureus strain isolated from the conjunctiva of a patient diagnosed of bacterial conjunctivitis, and were assayed on the basis of the zone of inhibition. One and two drops volume of the test drugs were inoculated (administered). Results for average inhibition zone for 1 and 2 drops volume was Levofloxacin (26.88 mm), Ciprofloxacin (25.0 mm), Tobramycin (12.5 mm), Gentamicin (7.5 mm) and Levofloxacin (33.12 mm), Ciprofloxacin (30.6 mm), Tobramycin $(19.3 \mathrm{~mm})$ and Gentamicin $(17.5 \mathrm{~mm})$ respectively with two drops having higher effect than one for Levofloxacin> Ciprofloxacin> Tobramycin> Gentamicin by way of order of susceptibility. The difference in the average zones of inhibition produced by equal volumes of gentamicin and tobramycin and that between ciprofloxacin and levofloxacin both not statistically significant $(\mathrm{p}>0.05)$. Fluoroquinolones showed greater zones of inhibition than the aminoglycosides significantly with levofloxacin more efficacious than ciprofloxacin. One drop prescription is thus recommended. However, if two drops must be prescribed perhaps due to severity of symptoms as the clinician may deem necessary, the drugs should be administered in divided doses. Antimicrobial sensitivity test should be conducted in cases of ocular bacterial infections caused by Staphylococcus aureus while treatment with fluoroquinolone especially levofloxacin eye drops as the first line treatment is also suggested
\end{abstract}

Keywords: Aminoglycosides; Fluoroquinolones; Eye drops; Staphylococcus aureus; Bacterial conjunctivitis; Inhibition zone

\section{Introduction}

The visual system is made up of three essential components: the optics of the eye which focuses an image of the environment on the retina, a system of nerves that transmit the image from the retina to the brain, and the visual cortex which processes the neural impulses [1]. The structure of the eye is highly organized and complex, reflecting the high degree of specialization that is required to support its function [2]. The vascularized nature of the conjunctiva coupled

\footnotetext{
* Corresponding author: Timothy Olugbenga Ogundeko

Department of Pharmacology and Therapeutics, College of Medicine and Health Sciences, Bingham University, Jos Campus Nigeria. 
with its exposure to dust, wind, heat and radiation easily gets infected. Staphylococcus aureus is the most common cause of bacterial conjunctivitis, blepharoconjunctivitis and keratoconjunctivitis [3]. Staphylococcus aureus is the leading cause of hospital-acquired infections by Gram-positive bacteria [4]. Ocular tissues are vulnerable to Staphylococcus aureus infection due to the exposed nature of the eyes. Among the pathogenic bacteria, Staphylococcus aureus is the most common cause of ocular infection by Gram-positive bacteria second only to Pseudomonas aeruginosa, causing ocular diseases such as conjunctivitis, blepharitis, blepharoconjunctivitis, corneal ulcer, dacryocystitis, orbital cellulitis and dacryoadenitis [5]. The future of modern medicine depends upon effective antibiotics [6].

Antibiotics are among the most frequently prescribed classes of medicines [7]. Antibiotic prescribing by physicians has gained due importance across the globe, mainly because of an increase in antibiotic usage, prevalence of infections, and drug resistances [8]. A report from a study in Nigeria by Anyanwu and Arigbe-Osula (2012) [9], stated that 63.3\% and $86.6 \%$ of prescriptions containing antibiotics, whereas the rate of antibiotic prescribing was higher for children aged 1-5 years compared to other groups combined ${ }^{10}$. According to Todar (2012) [10], a clinically useful antibiotic should be nontoxic to the host and without side effects, have a wide spectrum of activity, non-allergenic to the host, should not eliminate the normal flora of the host, of high bioavailability, chemically stable (long shelf life), inexpensive and easy to produce.

Aminoglycosides are natural or semisynthetic antibiotics derived from actinomycetes. They were among the first antibiotics to be introduced for routine clinical use and several examples have been approved for use in humans [11]. They are highly potent, broad-spectrum antibiotics that interfere with protein synthesis by selectively binding to the 30S [12]. Aminoglycoside antimicrobials were first introduced into clinical application in the middle and last half of the twentieth century primarily to treat severe infections caused by Gram-negative bacteria in animals [13], [14]. Examples include Gentamicin, Amikacin, Tobramycin, Streptomycin, Neo-Fradin, and Neomycin.

Fluoroquinolones (FQs) are counted among broad-spectrum antimicrobials and are used to treat genitourinary, respiratory, gastrointestinal, skin and soft tissue infections [15]. These medicines include ciprofloxacin (Cipro), gemifloxacin (Factive), levofloxacin (Levaquin), moxifloxacin (Avelox), norfloxacin (Noroxin), and ofloxacin (Floxin). Their mechanism of action is based on the drugs' ability to inhibit DNA gyrase and topoisomerase IV, and thus, DNA synthesis [16]. Resistance to fluoroquinolones in clinical isolates of $S$. aureus involves both mutational changes to the topoisomerases that reduce drug binding efficiency and elevated expression of endogenous efflux pumps [17].

This study aimed to ascertain the efficacy of 4 selected antibiotic eye drops against Staphylococcus aureus strain isolated from the conjunctiva of a patient diagnosed of bacterial conjunctivitis by way of their antibiotic classifications (Aminoglycosides and Fluoroquinolones).

\section{Material and methods}

\subsection{Study area, isolation and identification of test organism}

The organism was isolated from the conjunctival swab of a patient diagnosed of bacterial conjunctivitis. An inoculation loop sterilized in Bunsen flame was used to collect the organism, smeared and stained.

Staphylococcus aureus was isolated from the conjunctival swab of a student diagnosed of bacterial conjunctivitis in the Optometry Eye Clinic of Imo State University, Owerri, Nigeria and taken for microbiological analysis at the microbiology Laboratory in same institution. The isolate was cultured on nutrient agar for 24 hours. An inoculation loop sterilized in Bunsen flame was used to collect the organism, smeared, stained and identified based on macroscopic features. The result of the vitro growth response of the bacteria to the selected antibiotics as shown by the zones of inhibition (vertical (V) and horizontal (H) diameter) was obtained and recorded where one to two drops of the test drugs were considered. 


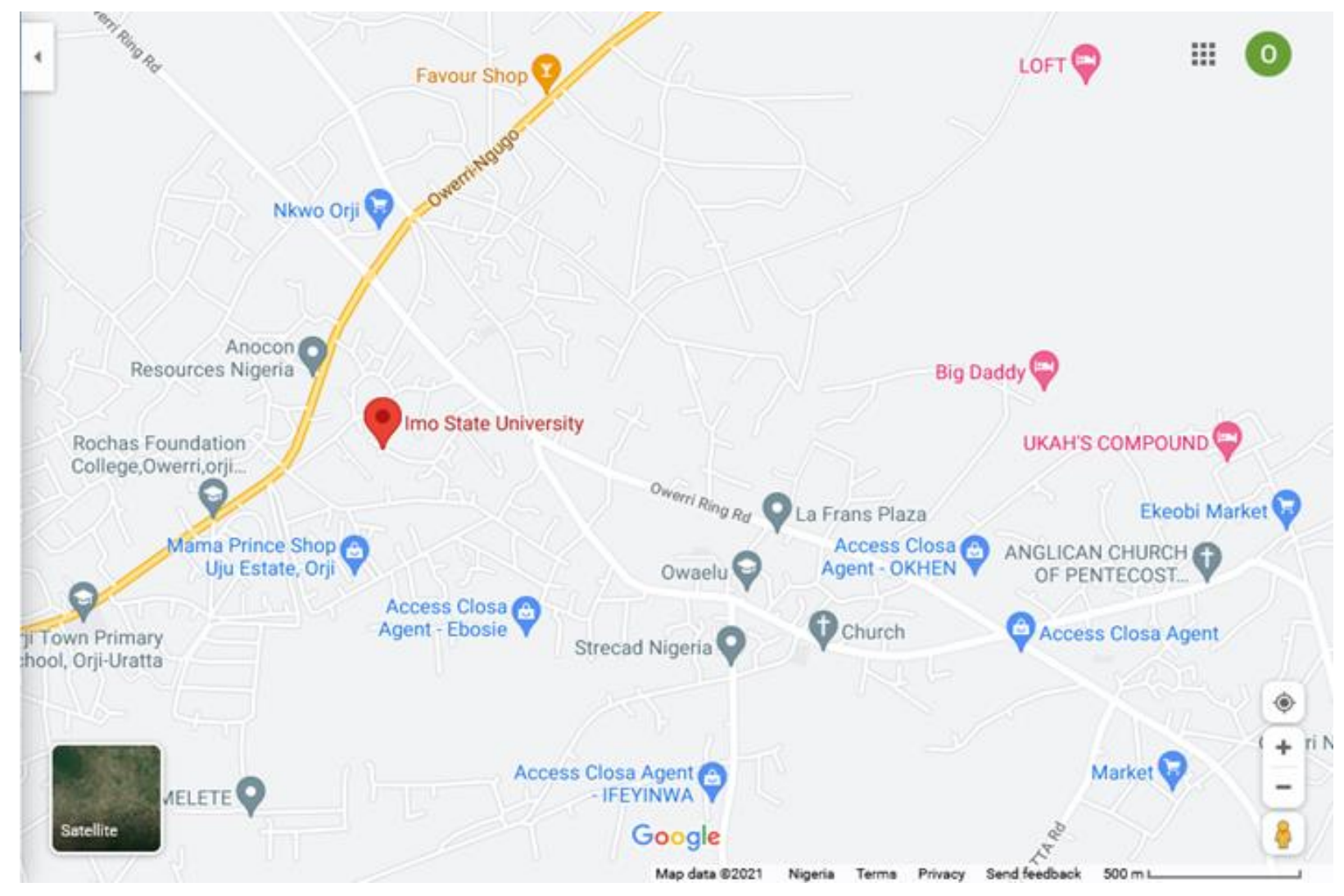

Figure 1 Google map of study area (Imo state University Owerri, Nigeria) [18].

\subsection{Test for antibacterial activity and Assay}

Antimicrobial activities were evaluated by the agar well diffusion method as described by Olurinola, (1996) [19] and Minimum Inhibitory Concentration (MIC) by Akinyemi et al., 2005 [20]. Antimicrobial susceptibility was tested on solid media (Nutrient agar) in petri plates the assay. Both Minimum Inhibitory Concentration (MIC) and the Minimum Bactericidal Concentration (MBC) were determined by serial micro dilution assay, suspension of culture for bacterial cells growth was done by preparing $2 \%$ Lauria Broth $(\mathrm{w} / \mathrm{v})$. The lowest concentrations without visible growth were defined as MICs as reported by Antra and Amla 2012. Microdilution method was used to determine the antibacterial activity of the test drugs against the test organism. Bacterial suspensions were adjusted with sterile saline to a concentration of $1 \times 10^{-7} \mathrm{CFU} / \mathrm{ml}$, while the prepared inoculum stored at $4^{\circ} \mathrm{C}[21]$.

\subsection{Test drugs}

The following drugs were purchased from pharmacy stores in Owerri metropolis, Imo State, Nigeria: QUIXIN ${ }^{\circledR}$ (Levofloxacin) ophthalmic solution $0.5 \%$ by Fidson Healthcare Plc, Nigeria, CILOXAN ${ }^{\circledR}$ (Ciprofloxacin) ophthalmic solution $0.3 \%$ by Alcon, Geneva, Switzerland, GENTALAB ${ }^{\circledR}$ (Gentamicin) ophthalmic solution $0.3 \%$ by Famzy Pharmaceuticals, Nigeria and TOBRAMYCIN ${ }^{\circledR}$ ophthalmic solution $0.3 \%$ by Bausch \& Lomb, Quebec, Canada.

\subsection{Statistical analysis}

This was performed using SPSS version 17.0 software (IBM-SPSS, Inc., Chicago, IL, USA). Data were compared using the Student's $t$-test. A probability $(p)$ value of $>0.05$ was considered statistically significant. 


\section{Results and discussion}

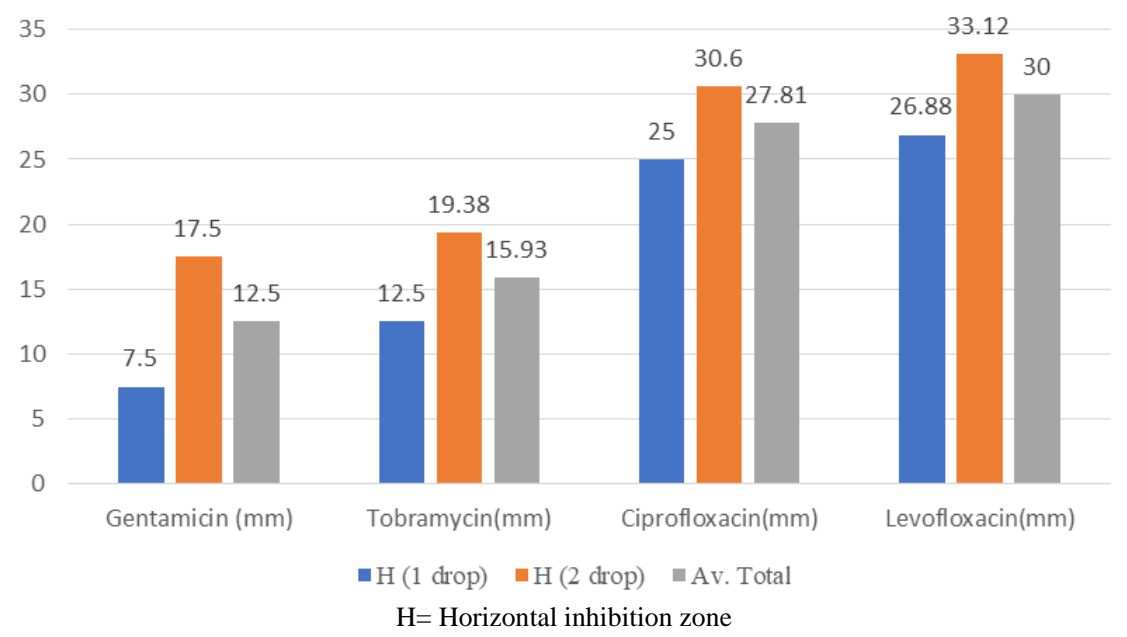

Figure 2 Horizontal Inhibition of various antibiotic on S. aureus

Figure 2 shows the trend of the horizontal $(\mathrm{H})$ meridians (diameters) inhibition zones by way of the efficacy of the test drugs against Staphylococcus aureus. For 1 drop of drug, it was Levofloxacin $(26.88 \mathrm{~mm})$, Ciprofloxacin $(25.0 \mathrm{~mm})$, Tobramycin $(12.5 \mathrm{~mm})$ and Gentamicin $(7.5 \mathrm{~mm})$ respectively. Two drops of drugs gave Levofloxacin $(33.12 \mathrm{~mm})$, Ciprofloxacin (30.6 mm), Tobramycin $(19.3 \mathrm{~mm})$ and Gentamicin $(17.5 \mathrm{~mm})$ respectively.

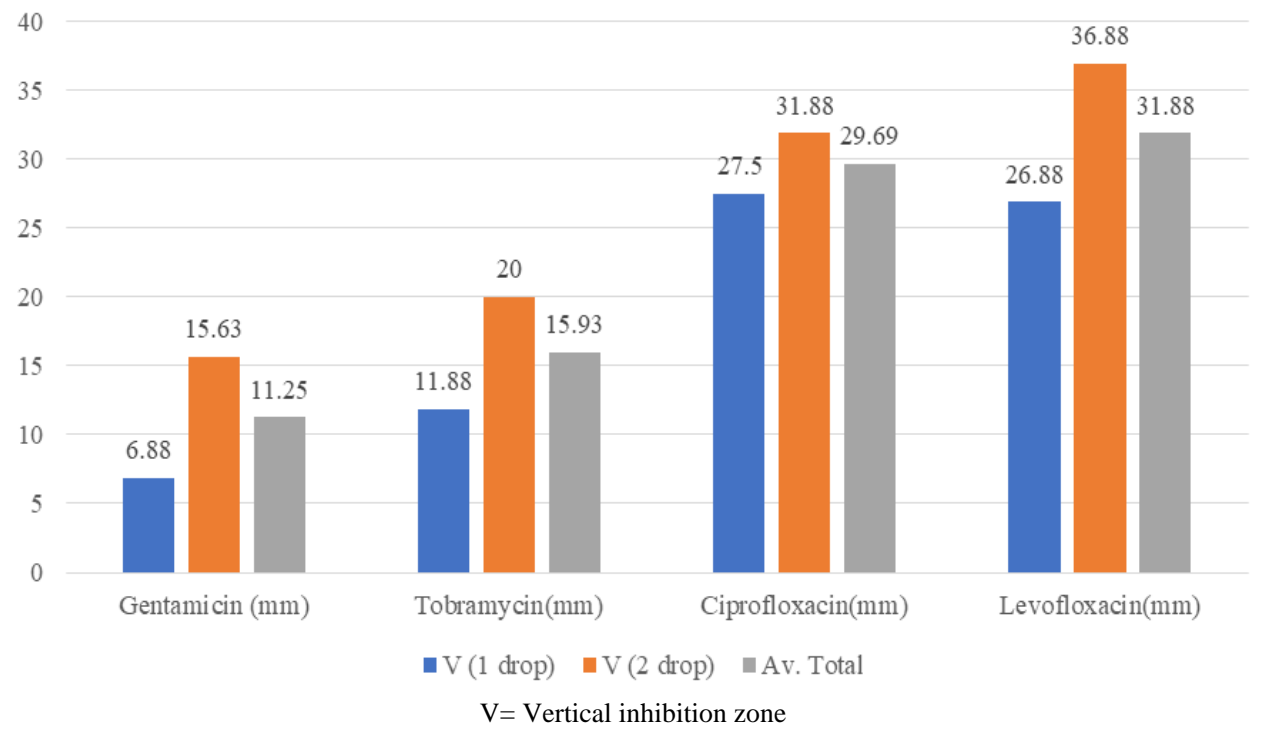

Figure 3 Vertical Inhibition of various antibiotic on S. aureus

Results for trend of the vertical (V) meridians (diameters) inhibition zones by way of the efficacy of the test drugs against Staphylococcus aureus had a slight change, for 1 drop of drug, it was Ciprofloxacin $(27.5 \mathrm{~mm})$, Levofloxacin $(26.88 \mathrm{~mm})$, Tobramycin $(11.88 \mathrm{~mm})$ and Gentamicin $(6.88 \mathrm{~mm})$ respectively, while two drops of drugs gave same trend like that of the horizontal zone with Levofloxacin $(36.88 \mathrm{~mm})$, Ciprofloxacin $(31.88 \mathrm{~mm})$, Tobramycin $(20.0 \mathrm{~mm})$ and Gentamicin (15.63 $\mathrm{mm}$ ) respectively - Figure 3.

Figures 2 and 3 however shows that 2 drops of the various test drugs were more efficacious than 1 drop. 
International Journal of Science and Research Archive, 2021, 02(02), 151-157

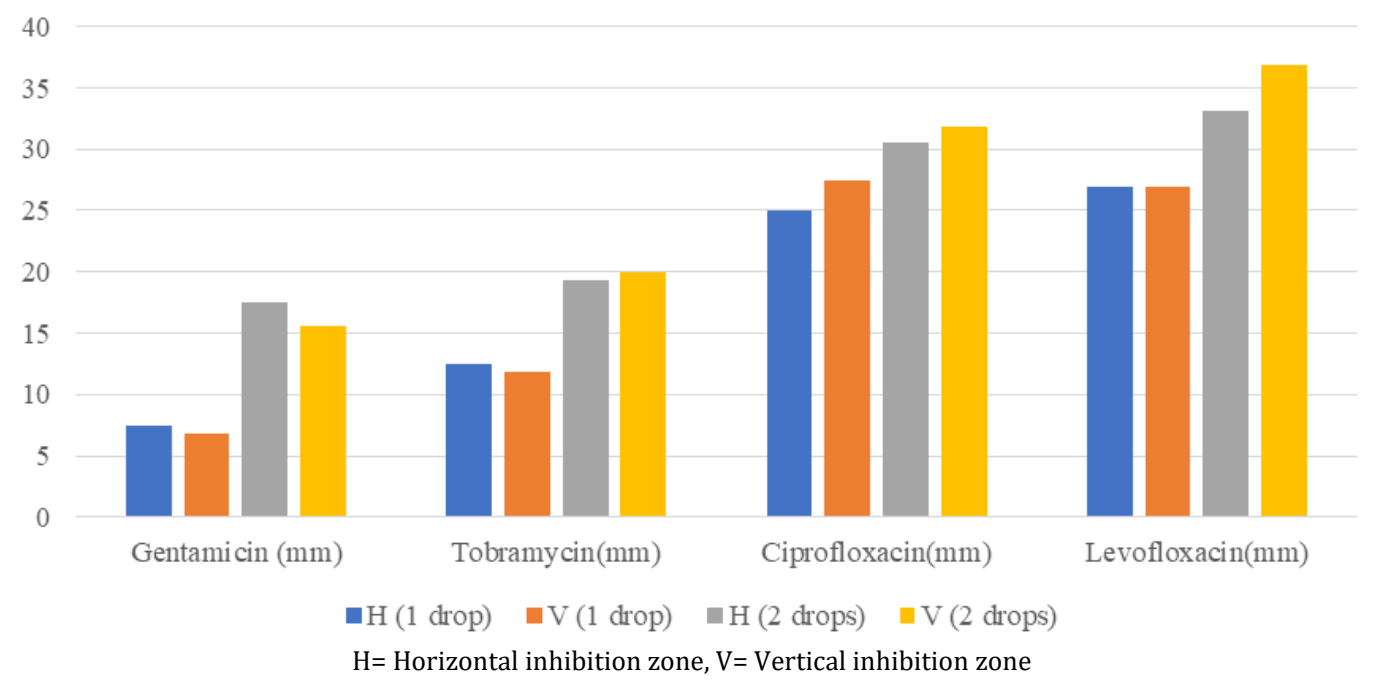

Figure 4 Average zones of inhibition caused by selected antibiotic on S. aureus

Tobramycin appear to be more effective against Staphylococcus aureus than gentamicin for equal volumes of drugs studied. Levofloxacin appears to be more effective against $S$. aureus than gentamicin for equal volumes of drugs used. For equal volumes of the drugs, the fluoroquinolones appear to more effective than aminoglycosides against Staphylococcus aureus in vitro - Figure 4.

Comparatively, in terms of the classes of the antibiotics, the zones of inhibition produced by 1 and 2 drops of aminoglycosides (gentamicin and tobramycin) measured in both vertical and horizontal meridians had averages of 5.75 $\mathrm{mm}, 13.25 \mathrm{~mm}, 9.75 \mathrm{~mm}$ and $15.75 \mathrm{~mm}$ respectively. Those for the fluoroquinolones (ciprofloxacin and levofloxacin) were $20.00 \mathrm{~mm}, 25.00 \mathrm{~mm}, 21.50 \mathrm{~mm}$ and $26.00 \mathrm{~mm}$ respectively - Figure 4 . There was no significant difference in the average efficacy of tobramycin and gentamicin as well as that of levofloxacin and ciprofloxacin against Staphylococcus aureus in vitro when two drops and one drop volumes are administered $(\mathrm{P}<2.447)$. Results further showed that the Staphylococcus aureus strain was resistant to one drop of tobramycin but susceptible to two drops of tobramycin. However, there was significant difference in the average efficacy of the fluoroquinolones and aminoglycosides against the randomly selected Staphylococcus aureus strain in vitro ( $>2.447)$.

Our study also showed that for equal volumes of the brands of aminoglycosides used, tobramycin proved to be more effective in inhibiting the growth of the Staphylococcus aureus than gentamicin although with no significant difference. Biswas et al., (2011) [22] listed tobramycin as potent against gram-negative bacteria whereas, Staphylococcus aureus is gram-positive. This highlights the need for further research to evaluate the efficacy of tobramycin against all strains of Staphylococcus aureus and the mechanism involved. Levofloxacin was more efficacious than ciprofloxacin against the $S$. aureus strain in vitro for equal volumes of the drugs. This supports the findings of (Davis \& Bryson, 1994) [23] and Graves et al., 2001 [24].

There was substantial difference in efficacy when one drop volume and two drops volume were compared for all the test drugs as 2 drops had higher effect than 1, following Levofloxacin $>$ Ciprofloxacin $>$ Tobramycin $>$ Gentamicin, in order words, considering the classes: fluoroquinolones > Aminoglycosides. However, one drop volume should be prescribed so as to avoid wastage considering the capacity of the conjunctival cul-de-sac. If two drops must be prescribed (perhaps due to severity of symptoms) as the clinician may deem necessary, then the drugs should be administered in divided doses.

\section{Conclusion}

Fluoroquinolones are more efficacious than the aminoglycosides against Staphylococcus aureus isolated from a conjunctivitis patient (in vitro). Two drops had higher effect than one, (Levofloxacin> Ciprofloxacin> Tobramycin> Gentamicin,). One drop should be prescribed so as to avoid wastage considering the capacity of the conjunctival cul-desac. If two drops must be prescribed (perhaps due to severity of symptoms) as the clinician may deem necessary, then the drugs should be administered in divided doses. 


\section{Compliance with ethical standards}

\section{Acknowledgments}

Authors wish to appreciate the various assistance by Megwas AU, Agu, GC and Akujobi AU of Imo State University, Owerri, Nigeria and Professor AP Bassi of Bingham University, Jos, Nigeria.

\section{Disclosure of conflict of interest}

All the authors hereby disclose no conflicts of interest/ competing Interests.

\section{Statement of informed consent}

Our study does not involve information about any individual; thus, statement of informed consent is not applicable.

\section{References}

[1] Chaudhry M. Refraction and lens prescription. 1st ed. New Dehli: CBS publishers Ltd. India; 2010.

[2] Caspi RR. A look at autoimmunity and inflammation in the eye. J Clin Invest. 1 Sep 2010; 120(9): 3073-3083.

[3] Nema HV, Ntin N. Textbook of ophthalmology. 5th ed. New Dehli: Jaypee brothers' publishers Ltd. India; 2008.

[4] Nsofor CA, Nwanekwu KEN, Nwachukwu IO. Basic microbiology. 1st ed. Owerri: Tonyben Publishers. Nigeria; 2013.

[5] Khurana AK, Khurana B. Comprehensive ophthalmology. 5th ed. New Dehli: New Age Publishers, India; 2012.

[6] So AD, Gupta N, Cars O. Tackling antibiotic resistance. BMJ Editorial. 2010; 340: c2071.

[7] Ramyil MSC, Ogundeko TO, Oyebode D. Antimicrobial sensitivity of Bacterial Isolates of patients attending the Bingham University Teaching Hospital, Jos, North Central, Nigeria. Bingham Journal of Medicine. 2020; (2)1: 3-7.

[8] Biswas M, Roy DN, Tajmim A, Rajib SS, Hossain M, Farzana F, Yasmen N. Prescription antibiotics for outpatients in Bangladesh: a cross-sectional health survey conducted in three cities. Ann Clin Microbiol Antimicrob. 2014; 13: 15.

[9] Anyanwu NC, Arigbe-osula ME. Pattern of antibiotic use in a tertiary hospital in Nigeria. European Journal of Hospital Pharmacy. April 2012; 19(2): 195.2-195.

[10] Todar K. Textbook of bacteriology. 2nd ed. Elsevier Inc. USA; 2012.

[11] Krause KM, Serio AW, Kane TR, Connolly LE. Aminoglycosides: An Overview. Cold Spring Harb Perspect Med. 2016 Jun; 6(6): a027029.

[12] Magnet S, Blanchard JS.2005. Molecular insights into aminoglycoside action and resistance. Chem. Rev. 2005; (105): 477-498.

[13] Schwarz S, Chaslus-Dancla E. Use of antimicrobials in veterinary medicine and mechanisms of resistance. Vet. Res. 2001; 32: 201-225.

[14] Maurin, M, Raoult D. Use of aminoglycosides in treatment of infections due to intracellular bacteria. Antimicrob. Agents Chemother. 2001; 45: 2977-2986.

[15] Liu HH. Safety Profile of the Fluoroquinolones. Drug Saf. 2010; 33: 353-369.

[16] Drlica K, Zhao X. DNA Gyrase, Topoisomerase IV, and the 4-Quinolones. 1997; 61: 377-392.

[17] Hooper DC, Jacoby GA. Mechanisms of drug resistance: quinolone resistance. Ann $N$ Y Acad Sci. 2015; 1354: 1231.

[18] Google Maps, 2021. ARU: Imo State University, Owerri, Imo State, Nigeria. Google Maps [online] Availablethrough: [Accessed 10 May 2021].

[19] Olurinola PF. A laboratory manual of pharmaceutical microbiology, Idu, Abuja, Nigeria. 1996; 69-105. 
[20] Akinyemi KO, Oladapo O, Okwara CE, Ibe CC, Fasure KA. Screening of crude extracts of six medicinal plants used in South - West Nigerian unorthodox medicine for antimethicillin resistant S. aureus activity. BMC Comp. Alt. Med. 2005; 5(6): 1-7.

[21] Antra Sen, Amla Batra. Evaluation of antimicrobial activity of different solvent extracts of medicinal plant: Melia azedarach l. International Journal of Current Pharmaceutical Research. 2012; 4: 2.

[22] Biswas NR, Viney G, Ashok D. Ocular therapeutics. 3rd ed. CBS publishers Ltd. New Dehli, India. 2011.

[23] Davis R, Bryson HM. Levofloxacin: a review of its antibacterial activities, pharmacokinetics and therapeutic efficacy. Journal Drugs. 1994; 47: 677-700.

[24] Graves A, Henry M, O’Brien TP, Hwang DG, Van Buskirk A, Trousdale MD. In vitro susceptibilities of bacterial ocular isolates to fluoroquinolones. Journal Cornea. 2001; 20: 301-305. 